\title{
Aneurysmal bone cyst of the spine
}

\author{
B. PRAKASH, A. K. BANERJI, AND P. N. TANDON \\ From the Department of Neurosurgery, All India Institute of Medical Sciences, \\ New Delhi-16, India
}

SUMMARY Four cases of aneurysmal bone cysts of the spine are described. Problems of nomenclature, pathogenesis, and treatment are discussed with reference to the literature. Complete excision is the treatment of choice whenever possible. However, subtotal excision and adequate spinal decompression followed by radiotherapy provide satisfactory results.

In 1942 Jaffe and Lichtenstein reviewed their pathological material relating to solitary bone cyst. They singled out two cases which showed a 'blown out' appearance radiologically and which contained fluid blood in the cyst. These lesions were called aneurysmal bone cyst. Lichtenstein's subsequent papers $(1950,1953)$ indicated the general acceptance of the pathological entity of aneurysmal bone cyst, and it had been clearly separated on histological as well as clinical grounds from giant cell tumours. Before Jaffe and Lichtenstein's (1942) review the lesion had been identified with a bewildering variety of names-namely, ossifying haematoma, subperiosteal giant cell tumour, aneurysmal giant cell tumour, haemorrhagic bone cyst, and expansile haemangioma (Sherman and Soong, 1957). There continue to be diverse opinions about the terminology (Kelpinger and Bucy 1961), aetiopathogenesis (Phelan, 1964), as well as treatment (MacCarty, Dahlin, Doyle, Lipscomb, and Pugh, 1961).

\section{CASE 1}

U.S., a 36 year old female, was admitted with complaints of pain in the neck and upper chest for three years and progressive weakness of the right arm. Examination revealed weakness of the shoulder, elbow, and wrist on the right side with tenderness over C5-6 vertebral spinous processes.

Radiological examination revealed ballooning of the lamina of the sixth cervical vertebra. A lumbar route myelogram demonstrated a block at the same level suggestive of an extradural lesion (Fig. 1). At surgery the spinous process and the lamina of the
C6 vertebra were found to be enlarged and spongy and filled with reddish material resembling vascular granulation tissue. Laminectomy with removal of all posteriorly lying abnormal tissue was performed to achieve adequate decompression. Postoperatively the patient received a course of radiotherapy.

Biopsy revealed extensive destruction of the bony trabeculae which were replaced by large cavernous spaces lined by endothelium. Intermixed with blood in these spaces were a large number of giant cells with 4 to 10 nuclei. There was some evidence of new bone formation in some parts of the lesion.

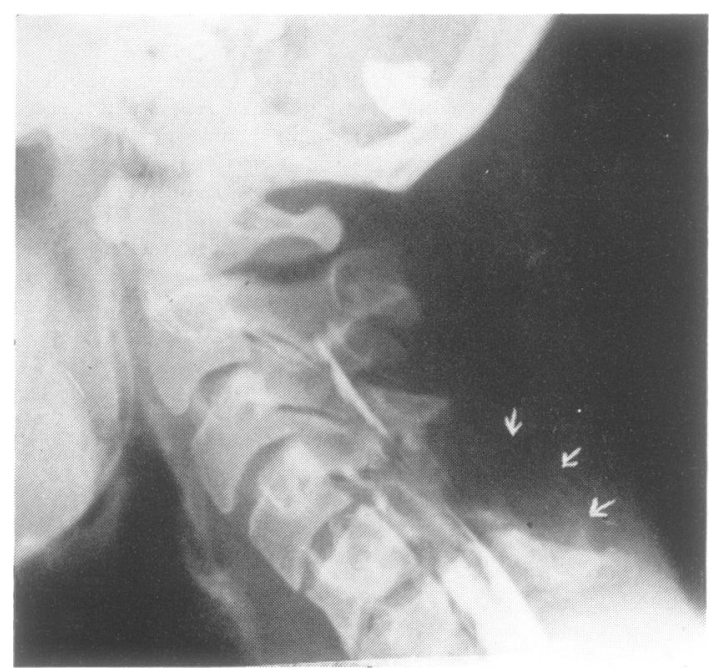

FIG. 1. Case 1. Myelogram (lateral view) showing block at lower level of C5 vertebra with complete destruction of neural arch and spinous process. 


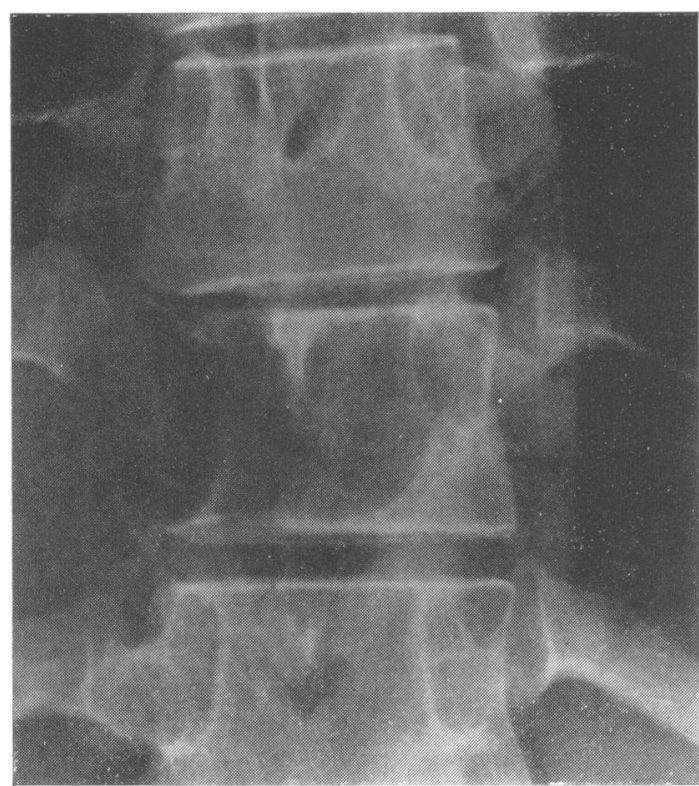

FIG. 2. Case 2. Plain radiograph of the spine (AP view) showing erosion of the pedicle of D4 vertebra.

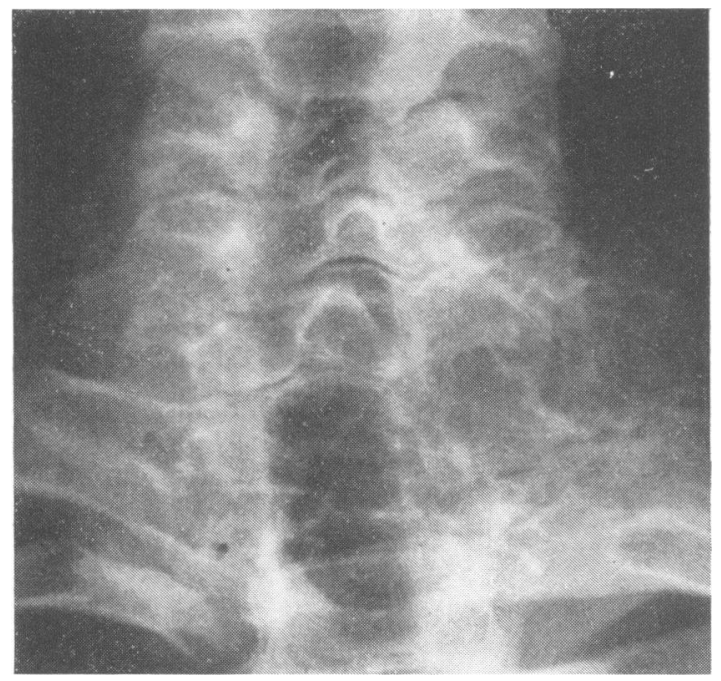

FIG. 3. Case 3. Plain radiograph of the spine ( $A P$ view) showing cystic lesion of the posterior neural arch.

COMMENTS In view of the long history and tenderness of the spine a clinical diagnosis of tuberculous spine was made. The surgical excision being subtotal, postoperative radiotherapy was given. On follow-up examination six years after surgery the patient was completely symptom free.

\section{CASE 2}

M.S., a 48 year old female, was admitted with complaints of tingling and numbness of both lower limbs for three months and progressive paraparesis of 20 days' duration. Physical examination revealed a spastic paraparesis with a sensory level at D6 dermatome.

Anteroposterior radiographs of the dorsal spine revealed absence of the pedicles of the 4 th dorsal vertebra (D4) (Fig. 2). A lumbar route myelogram demonstrated an extradural type of block at the upper border of D5 vertebra. At operation the spinous process and lamina of D4 vertebra were found to be replaced by spongy vascular tissue which extended to the dura mater. The pedicles were involved on both sides. The involved posterior neural arch was excised. The vertebral body which was also involved was not touched. Postoperatively the patient was given a course of radiotherapy $(1,900 r)$. She made a complete neurological recovery within three weeks.

Biopsy was reported as follows: 'There were large cavernous pools of blood with indefinite and illdefined endothelial lining. Scattered in irregular groups and intermixed with blood-filled spaces were large sheets of giant cells. These contained nuclei which varied from two to over 50 , resembling osteoclastic type of giant cell. There was scant stroma made up of spindle shaped cells'.

COMMENT The radiological diagnosis was probably missed because of the inability to obtain a good lateral view of the affected region. As the excision was considered to be subtotal, postoperative radiotherapy was administered. The patient was asymptomatic at the last follow-up four years after surgery.

\section{CASE 3}

O.S., a male aged 17 years, was admitted with progressive quadriparesis of nine months' duration and a progressively increasing swelling over the nape of the neck of six months' duration. Physical examination revealed a moderately tender bony swelling about $3 \mathrm{~cm} \times 3 \mathrm{~cm} \times 1 \mathrm{~cm}$ in relation to the spinous processes of $\mathrm{C} 7$ and $\mathrm{D} 1$ vertebrae. Neurological examination revealed a flaccid paresis of the left arm with complete loss of power in the legs, which were spastic. There was a sensory level at D2 dermatome. The urinary bladder was spastic.

A plain radiograph of the cervical spine revealed an expanding cystic lesion of the posterior neural arch, including the spinous process of D1 vertebra. The lesion extended into the vertebral body (Fig. 3). A radiological diagnosis of aneurysmal bone cyst was made and to define the upper border of the cord 


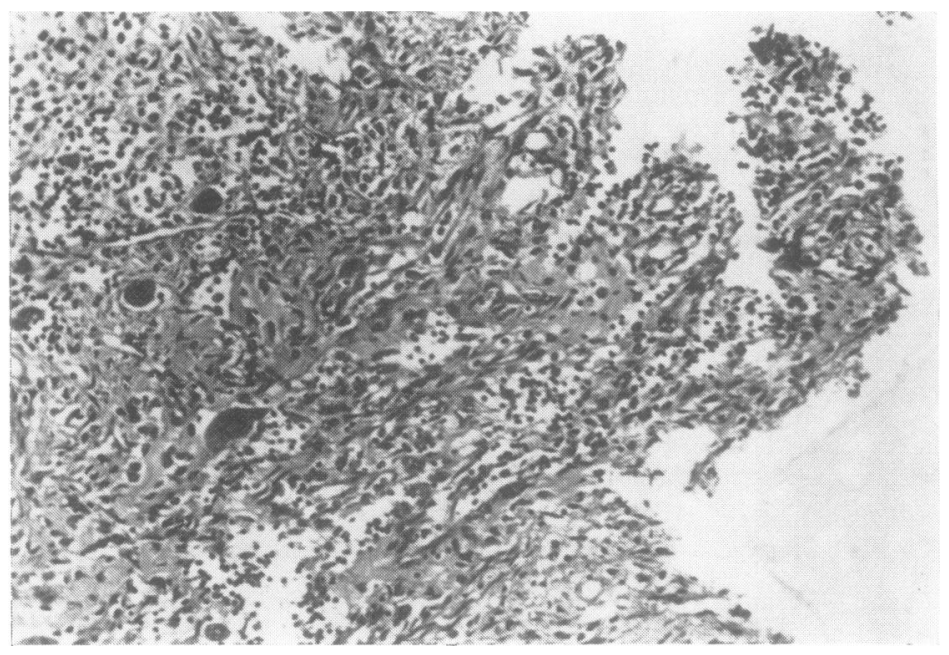

FIG. 4. Case 3. Microphotograph showing the thin-walled vascular channels and multinucleated giant cells. $H$ and $E$, $\times 160$.

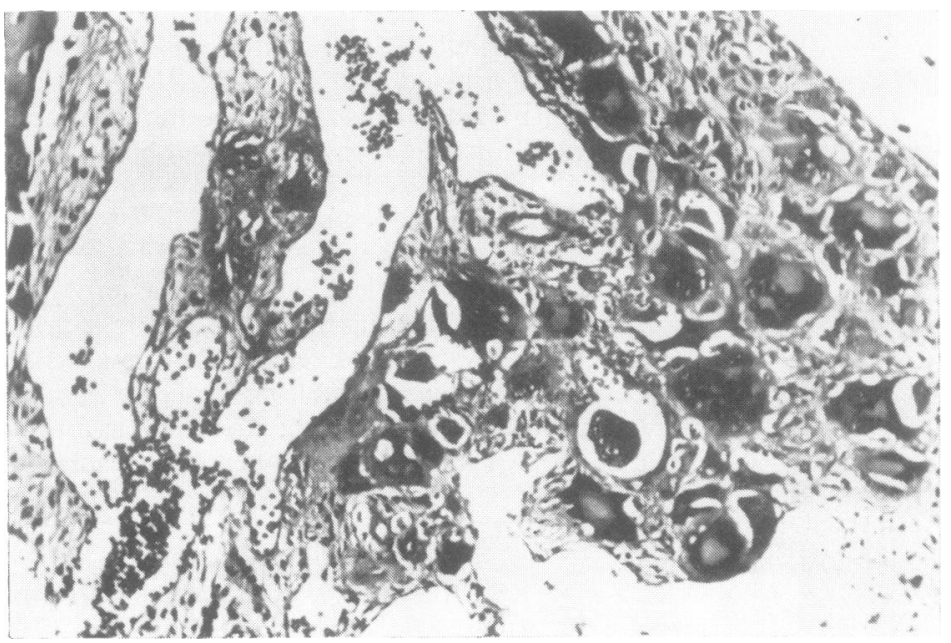

FIG. 5. Case 3. Microphotograph showing cystic vascular spaces filled with blood and intermixed with multinucleated giant cells. $H$ and $E, \times 160$.

compression a cisternal route myelogram was done. This revealed an extradural type of block at C7 vertebra. The affected posterior neural arch of D1 vertebra was excised with the extradural extension of the tumour. The findings at surgery were characteristic of an aneurysmal bone cyst. Postoperatively the patient received radiotherapy $(1,880 r)$. There was some improvement in the power of the left upper limb, but no recovery was observed in the lower limbs at the time of discharge three weeks after surgery.

Biopsy was reported as follows: 'Extensive destruction of the bony trabeculae, which in many places were completely replaced by large distended cavernous spaces lined by endothelium and filled with blood. Closely intermingled with such vascular spaces were large masses of giant cells which appeared syncitial in character. The nuclei showed evidence of moderately severe pleomorphism but no mitotic figures were seen. The surrounding bony trabeculae showed evidence of remodelling as evidenced by active osteoblastic activity and new bone formation.' Figures 4 and 5 represent the typical picture of an aneurysmal bone cyst. 
COMMENTS This patient presented with a swelling overlying a spinous process and progressive quadriparesis which in India would be clinically suggestive of spinal caries. Radiological examination established the correct diagnosis preoperatively. As expected there was only partial neurological recovery due to the dense involvement preoperatively. This patient has been lost to follow up.

\section{CASE 4}

G.P., a 20 year old male, was admitted with progressive paraparesis for four months. Examination revealed paravertebral fullness in the lower dorsal region with tenderness of the D10 vertebra. Neurological examination revealed a complete spastic paraplegia with a sensory level at D10 dermatome.

Radiographs of the dorsal spine revealed a ballooning of spinous process and absence of the pedicle on the right side of the D10 vertebra. At operation the spinous process, lamina, and pedicles of D10 vertebra were found to be characteristically enlarged, spongy, and full of vascular reddish-brown tumour tissue. Postoperatively the patient was given a course of radiotherapy $(1,100 r)$. During his hospital stay the patient started to show recovery of power in the legs. Six months after discharge from the hospital he was neurologically asymptomatic.

Biopsy revealed extensive replacement of bony tissue by large aneurysmally dilated spaces distended with blood. Endothelial lining was recognizable in only some parts. Numerous giant cells were scattered irregularly in the tissue. There was much variation in the size, shape, and number of the nuclei, which varied from four to numerous. Stroma showed evidence of old haemorrhage and extensive new bone formation.

COMMENTS This case was again diagnosed clinically as tuberculous spine because of the paravertebral fullness, tenderness of the spine, and progressive paraparesis. However, after radiological examination, a preoperative diagnosis of aneurysmal bone cyst was made.

\section{DISCUSSION}

The nomenclature of the lesion has been the subject of some controversy (Taylor, 1956; Keplinger and Bucy, 1961). It is generally agreed that a giant cell tumour is in a completely separate category and that there is no reason to include aneurysmal bone cysts under the title of giant cell tumour as advocated by Keplinger and Bucy (1961).

On gross histological examination an aneurys- mal bone cyst is easily differentiated from a giant cell tumour. An aneurysmal bone cyst contains cystic spaces filled with blood, whereas the giant cell tumour rarely shows cysts. Under the microscope the aneurysmal bone cyst consists of cavernous spaces lined with indistinct endothelium with giant cells situated in the matrix. These cells are small to moderate in size with few nuclei, whereas the true giant cell tumour shows bigger cells containing large numbers of nuclei, and there is always evidence of newly formed blood vessels.

By definition, an aneurysm is a sac or space formed by widening or extension of a vessel containing blood or clot. The aneurysmal components of an aneurysmal bone cyst are really separate cystic spaces and are not extensions of an artery or vein and so the term 'aneurysmal' is a misnomer. For this reason, Taylor (1956) suggested the term haemangiomatous bone cyst, and we feel that this is more appropriate bearing in mind the arteriovenous shunt mechanism which has been demonstrated in this lesion (Lindbom, Soderberg, Spjut, and Sunngirst, 1961).

Nearly three decades after its first description the pathogenesis of aneurysmal bone cyst is still not well established. These cysts constituted $1.5 \%$ of all bone tumours seen at the Mayo Clinic, and nine of 61 cases involved the vertebrae (MacCarty et al., 1961). After the long bones the spine is the commonest site to be involved (Subramaniam and Mathias, 1962).

The majority of cases reported were below the age of 30 years (Donaldson, 1962). The youngest case reported was 2 years old (Keplinger and Bucy, 1961), and the oldest was 61 years (Sherman and Soong, 1958). Our cases ranged from 18 years to 48 years, with two males and two females. In the reported cases there appears to be an equal incidence in both the sexes, even though individual reports suggest a female (Poppen, 1961) or male (Lichtenstein, 1951) preponderance. In three out of our four cases the lesion was situated in the dorsal spine, while a cervical vertebra was involved in the fourth. In the Mayo Clinic series (MacCarty et al., 1961), four were cervical, two sacral, and one each in the dorsal and lumbar regions.

In none of our cases was there any history of injury preceding the onset of symptoms. All the 
eight cases of Poppen (1961) had a history of injury preceding the onset of symptoms by six months to a year. Of the four cases of Subramaniam and Mathias (1962), two gave a history of injury. Lichtenstein (1950) felt that injury was unlikely to play any part in the aetiology of this lesion. However, Cone (1928) had suggested the possibility of the cyst being an organizing subperiosteal haematoma and in that light the history of injury may have some importance.

One of our cases had presented with local pain radiating along the roots, while another patient had presented with local pain only. In two cases there was no history of local pain. Eight of the nine cases reported by MacCarty et al. (1961) had presented in this way. Local pain, though common, is not invariable.

In four of the nine cases of MacCarty et al. (1961) there was a palpable local swelling, while in one there was a questionable swelling and another had a swelling palpable per rectum. A definite swelling was palpable in one of our cases and it was doubtful in another. Clinical evidence of a paravertebral swelling and neurological deficit in a patient in India raises the suspicion of tuberculosis of the spine. All our patients had a well-marked level of neurological involvement which corresponded fairly well with the site of the bony lesion and the operative findings.

Radiological examination revealed a typical cystic lesion of spinous processes and laminae in three of our cases. In one case affecting the fourth dorsal vertebra an anteroposterior radiograph had revealed absence of the pedicles. A satisfactory lateral view had not been obtained. In view of the operative findings a lateral radiograph would have demonstrated the lesion involving the lamina and the spinous process. Radiological evidence of involvement of the vertebral body was seen in one of our cases. Donaldson (1962) found the posterior elements of the vertebra to be commonly involved. Sherman and Soong (1957), observed the laminae to be involved in every instance, as was confirmed by our cases. Involvement of the vertebral body is described usually as an extension of the process beginning posteriorly and similarly involvement of adjacent vertebrae has also been described (MacCarty et al., 1961).

The natural history of aneurysmal bone cyst has been traced radiologically by Sherman and
Soong (1957). The lesion begins as an eccentric area of rarefaction with little or no internal structure. Within a few months the rarefied area matures into a cyst with well-defined borders and internal septa. After maturation the cyst grows at an unpredictable rate, unrelated to the age or site of the lesion. A period of latency may be followed by reactivity. Spontaneous regression or transformation from a full-blown lesion to a calcified or ossified mass may also be seen. Once calcified the lesion was not observed to recur.

A subtotal excision was done in all our cases. On surgical exposure the lesion appeared as a distended vertebral lamina and/or spinous process. The lesion consisted of multiple cystic cavities as well as extra-osseous extradural vascular soft tissue compressing the cord. All the grossly abnormal tissue presenting posteriorly was removed, though no attempt was made to remove the diseased tissue totally. All cases except case 2 showed a moderately quick recovery of spinal cord function. Case 2 was already quadriplegic with complete loss of motor and sensory function in the lower limbs. All our cases received postoperative radiotherapy, in doses varying from 800 to $2,000 r$. Postoperative recovery was maintained at the time of follow-up varying from one and a half to seven years.

The therapeutic approach to aneurysmal bone cyst has undergone considerable changes since the lesion was first described. Lichtenstein (1953) suggested that biopsy should be followed by radiotherapy $(2,000 r)$. Regen and Haber (1957) treated a case of aneurysmal bone cyst of the body of the 3rd cervical vertebra, which presented as a retropharyngeal mass, by biopsy and radiotherapy with a good result. Kelpinger and Bucy (1961) noted extensive calcification after radiotherapy and commented that, if the spinal canal is insufficiently decompressed, such calcification may itself cause compression. They advocated laminectomy and extensive decompression followed by radiotherapy. This was the common method of treatment used in most centres. MacCarty et al. (1961), however, felt that in view of the essentially benign nature of the lesion, and a risk of malignancy after radiotherapy, complete excision should be used when possible. As the posterior elements of the vertebrae are commonly involved (Donaldson, 1962), a complete excision is often possible by 
laminectomy alone. In lesions of the vertebral body an anterior approach may be used. In extensive involvement of a vertebral body curettage and use of homologous bone chips was advised by MacCarty et al. (1961). Complete recovery after surgical excision has also been reported by Verbiest (1965) and by McArthur and Fisher (1966).

Recurrence of the lesion has been noted after partial excision and radiotherapy (MacCarty et al., 1961), and also after radiotherapy alone (Sherman and Soong, 1957). The ideal treatment for all accessible lesions appears to be complete excision (MacCarty et al., 1961; Verbiest, 1965). Radiotherapy is resorted to after surgical decompression of the spinal canal only if only partial excision has been achieved.

\section{REFERENCES}

Cone, S. M. (1928). Ossifying hematoma. Journal of Bone and Joint Surgery, 10, 474-482.

Donaldson, W. F., Jr. (1962). Aneurysmal bone cyst. Journal of Bone and Joint Surgery, 44A, 25-40.

Jaffe, H. A. (1962). Discussion of paper by W. F. Donaldson, Jr., Journal of Bone and Joint Surgery, 44A, 40.

Jaffe, H. L., and Lichtenstein, L. (1942). Solitary unicameral bone cyst, with emphasis on roentgen picture, the pathological appearance and the pathogenesis. Archives of Surgery, 44, 1004-1025.
Keplinger, J. E., and Bucy, P. C. (1961). Giant-cell tumors of the spine. Annals of Surgery, 154, 648-661.

Lichtenstein, L. (1950). Aneurysmal bone cyst. A pathological entity commonly mistaken for giant-cell tumor and occasionally for hemangioma and osteogenic sarcoma. Cancer, 3, 279-289.

Lichtenstein, L. (1953). Aneurysmal bone cyst: further observations. Cancer, 6, 1228-1237.

Lindbom, A., Soderberg, G., Spjut, H. L., and Sunnqvist, $\mathbf{O}$. (1961). Angiography of aneurysmal bone cyst. Acta Radiologica, 55, 12-16.

McArthur, R. A., and Fisher, R. G. (1966). Aneurysmal bone cyst involving the vertebral column. A case report. Journal of Neurosurgery, 24, 772-776.

MacCarty, C. S., Dahlin, D. C., Doyle, J. B., Jr., Lipscomb, P. R., and Pugh, D. G. (1961). Aneurysmal bone cysts of the neural axis. Journal of Neurosurgery, 18, 671-677.

Phelan, J. T. (1964). Aneurysmal bone cyst. Surgery, Gynecology, and Obstetrics, 119, 979-983.

Poppen, J. L. (1961). Discussion: Giant cell tumors of the spine. (By J. E. Keplinger, and P. C. Bucy.) Annals of Surgery, 154, 648-661.

Regen, E. M., and Haber, A. (1957). Giant-cell tumor of cervical vertebra with unusual symptoms. Reprint of a case and review of the literature. Journal of Bone and Joint Surgery, 39A, 196-200.

Sherman, R. S., and Soong, K. Y. (1957). Aneurysmal bone cyst: its roentgen diagnosis. Radiology, 68, 54-64.

Subramaniam, C. S. V., and Mathias, P. F. (1962). Aneurysmal bone cyst. Journal of Bone and Joint Surgery, 44B, 93101.

Taylor, F. W. (1956). Aneurysmal bone cyst. A report of three cases. Journal of Bone and Joint Surgery, 38B, 293300 .

Verbiest, H. (1965). Giant-cell tumours and aneurysmal bone cysts of the spine. Journal of Bone and Joint Surgery, 47B, 699-713. 